\title{
A new Early Cretaceous flea from China
}

Yanjie Zhang, Chungkun Shih, Alexandr P. Rasnitsyn, Dong Ren, and Taiping Gao Acta Palaeontologica Polonica 65 (1), 2020: 99-107 doi:https://doi.org/10.4202/app.00680.2019

Fleas are highly specialized holometabolic insects. So far, only 16 species of fossil fleas in five families have been reported due to the rare fossil records. At present, the earliest flea fossils are reported from the Middle Jurassic Jiulongshan Formation of Northeastern China. The descriptions of these earliest species pushed back the origin of Siphonaptera by at least 40 million years. It is generally accepted that saurophthirids are the "transitional" taxa from stem Jurassic fleas to living crown groups. Herein, we described a new "transitional" flea species, Saurophthirus laevigatus Zhang, Shih, Rasnitsyn, and Gao sp. nov., from the Lower Cretaceous Yixian Formation of Northeastern China, assigned to Saurophthiridae. The new species provides new evidence to support saurophthirids as a "transitional" group. Sexual dimorphism suggests significant differences in biology of opposite sexes in Saurophthirus. Analysis of described Mesozoic species demonstrates the body size reduction from the Middle Jurassic to the Early Cretaceous. Smaller body size was likely advantageous in reducing the probability of being detected and removed by the host and in minimizing flea's demand for blood intake and energy input, indicating the adaptation of the ectoparasitic lifestyle of fleas in their early stage of evolution.

Key words: Insecta, Siphonaptera, Saurophthiridae, Saurophthirus, ectoparasitic insects, compression fossils, Mesozoic, Asia.

Yanjie Zhang [15011010331@163.com], Chungkun Shih [chungkun.shih@gmail.com

], Dong Ren [rendong@mail.cnu.edu.cn], and Taiping Gao [tpgao@cnu.edu.cn

] (corresponding author), College of Life Sciences, Capital Normal University, 105 Xisanhuanbeilu, Haidian District, Beijing 100048, China;

Academy for Multidisciplinary Studies, Capital Normal University, 105

Xisanhuanbeilu, Haidian District, Beijing 100048, China. Chungkun Shih [chungkun.shih@gmail.com ], Department of Paleobiology, National Museum of Natural History, Smithsonian Institution, Washington, DC 20013-7012, USA. Alexandr P. Rasnitsyn [alex.rasnitsyn@gmail.com], Palaeontological Institute, Russian Academy of Sciences, Moscow 117997, Russia; Natural History Museum, Cromwell Road, London SW7 5BD, UK. 
This is an open-access article distributed under the terms of the Creative Commons

Attribution License (for details please see creativecommons.org), which permits unrestricted use, distribution, and reproduction in any medium, provided the original author and source are credited.

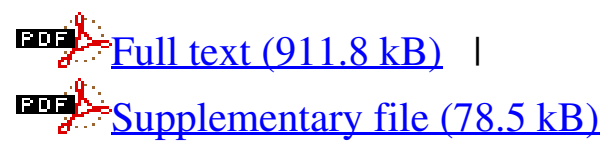

